\title{
Yokukansan improves behavioral and psychological symptoms of dementia by suppressing dopaminergic function
}

This article was published in the following Dove Press journal:

Neuropsychiatric Disease and Treatment

15 March 2016

Number of times this article has been viewed

\section{Kenji Takeyoshi ${ }^{1,2}$ \\ Masatake Kurita ${ }^{1-3}$ \\ Satoshi Nishino ${ }^{2,3}$ \\ Mika Teranishi' \\ Yukio Numata ${ }^{2}$ \\ Tadahiro Sato ${ }^{2}$ \\ Yoshiro Okubo'}

'Department of Psychiatry and Behavioral Science, Graduate School of Medicine, Nippon Medical School, Bunkyo-ku, Tokyo, ${ }^{2}$ Sato Hospital, Koutokukai, Nanyo, Yamagata, ${ }^{3}$ Department of Cellular Signaling, Graduate School of Pharmaceutical Sciences, Tohoku University, Sendai, Miyagi, Japan
Correspondence: Masatake Kurita Sato Hospital, Koutokukai, 948-I Kunugizuka, Nanyo, Yamagata 999-2221, Japan

$\mathrm{Tel}+81238403170$

Fax +8I 238432076

Email kurita@koutoku.or.jp
Abstract: Although three drugs, risperidone, yokukansan, and fluvoxamine, have shown equal efficacy in treating behavioral and psychological symptoms of dementia (BPSD) in our previous study, their mechanisms of action are different from one another. Monoamines have attracted attention for their key roles in mediating several behavioral symptoms or psychological symptoms through synaptic signaling. We aimed to clarify the monoamines changed by treatment with each drug in patients with BPSD. The main purpose of this study was to determine whether plasma levels of catecholamine metabolites are correlated with pharmacological treatments. This was an 8-week, rater-blinded, randomized, flexible-dose, triple-group trial. In total, 90 subjects were recruited and subsequently three different drugs were allocated to 82 inpatients with BPSD. We examined BPSD data from patients who completed 8 weeks of treatment. Eventually, we analyzed 42 patients (yokukansan: 17; risperidone: 9; fluvoxamine: 16). Homovanillic acid, a metabolite of dopamine, and 3-methoxy-4-hydroxyphenylglycol, a metabolite of noradrenaline, in their plasma were analyzed by high-performance liquid chromatography with electrochemical detection. All three drugs showed equal significant efficacy between baseline and study endpoint. By contrast, biomarkers showed mutually different changes. Patients in the yokukansan group had significantly decreased plasma homovanillic acid levels from baseline. Conversely, patients in the risperidone and fluvoxamine groups exhibited no significant changes in plasma homovanillic acid levels from baseline. Yokukansan contains geissoschizine methyl ether, which is known to have a partial agonist effect on dopamine D2 receptors. An improvement in BPSD condition with the intake of yokukansan is suggested to occur through a suppressed dopaminergic function, which is similar to the effect of aripiprazole.

Keywords: herbal medicine, MHPG, homovanillic acid, HVA, noradrenaline, BPSD

\section{Introduction}

The term behavioral and psychological symptoms of dementia (BPSD) is used to cover a range of non-cognitive disturbances including anxiety, depression, irritability, aggression, agitation, eating disorders, and inappropriate social or sexual behaviors. ${ }^{1,2}$ BPSD is seen in about $90 \%$ of patients with dementia. ${ }^{3}$ Although it is difficult to improve cognitive function - the core symptom of dementia - through pharmacological treatments, BPSD is reported to improve after certain pharmacological treatments, such as by herbal medicines, ${ }^{4-9}$ antipsychotic drugs, ${ }^{10}$ and antidepressants. ${ }^{9,11}$ Lanari et al proposed that the pathophysiology of BPSD is related to an imbalance among different neurotransmitters, namely acetylcholine, serotonin, dopamine, and noradrenaline. ${ }^{12}$

Yokukansan was developed in 1555 by Xue Kai, which is a traditional medicine - the so-called kampo medicine - in Japan. It has been approved by the Ministry 
of Health, Labour and Welfare of Japan as a remedy for neurosis, insomnia, and irritability in children. The clinical efficacy of yokukansan in the treatment of BPSD has been demonstrated not only in open studies ${ }^{5-7}$ but also in a randomized cross-over study. ${ }^{13}$ The neuropharmacological properties of yokukansan have been suggested to include its serotonin receptor agonistic effects. ${ }^{14}$ Currently, the most common drug therapy for BPSD is antipsychotics. ${ }^{15}$ However, adverse reactions such as deterioration of cognitive function, extrapyramidal symptoms, and gait disturbance limit the use of atypical antipsychotics in the treatment of these symptoms. ${ }^{16}$ The neuropharmacological properties of numerous antipsychotics are primarily exerted through dopamine antagonism. ${ }^{17}$ Previously, we prescribed a selective serotonin reuptake inhibitor (SSRI), fluvoxamine, for the treatment of BPSD in three patients with dementia and reported its efficacy. ${ }^{18}$ Additionally, randomized studies have demonstrated the efficacy of fluvoxamine ${ }^{9}$ and citalopram ${ }^{11}$ for BPSD. Fluvoxamine is an SSRI that has been available as an antidepressant since 1983 in many countries. The neuropharmacological properties of fluvoxamine have been suggested to include its serotonin reuptake inhibitor effects. However, no current hypotheses can sufficiently explain the pathophysiological and neurobiological basis of BPSD.

Despite numerous advances toward understanding the pharmacological treatments of BPSD, the outcomes for the underlying mechanisms remain poor because of the limited understanding of BPSD etiology. Clarification of the neurobiological basis in the etiology of BPSD is an important issue from the viewpoint of clinical strategy. In particular, monoamines have attracted attention for their key roles in mediating several behavioral symptoms or psychological symptoms through synaptic signaling, all of which are thought to be involved in the pathophysiology of psychiatry. Our previous report ${ }^{9}$ has shown the efficacy and tolerability of risperidone, yokukansan, and fluvoxamine for BPSD. Therefore, we focused on the biological changes as the secondary analysis in this study. There is currently no biomarker that can serve as an objective index for evaluating BPSD. The main purpose of this study was to determine whether plasma levels of catecholamine metabolites are correlated with pharmacological treatments.

\section{Materials and methods Study design}

This was an 8-week, rater-blinded, randomized, flexible-dose, triple-group trial. Participants with dementia underwent treatment for BPSD in hospitals because their home caregivers, and even nursing homes or similar institutions, could no longer manage their BPSD. Ninety patients were selected from the inpatients with BPSD at Sato Hospital, a psychiatric hospital in Nanyo (Yamagata, Japan). Data were collected between January 2009 and August 2010 and registered in the UMIN Clinical Trials Registry (identifier: UMIN000006146). This study relates to the secondary analysis of a previous study. ${ }^{9}$

Patients or their legally authorized representatives provided written informed consent before entering the trial, after receiving a detailed explanation of the procedures and possible adverse events. The study protocol was approved by the Ethics Committees of Sato Hospital and Tohoku University (Sendai, Miyagi, Japan). The study was conducted in accordance with the Declaration of Helsinki. Patients who met the inclusion and exclusion criteria and who were able to provide written informed consent before entering this study were accepted. According to the requirements of the ethics committees, written informed consent was obtained from the patients themselves participating in the study as much as possible. No important changes to the methods were made after trial commencement, and no interim analyses or stopping guidelines were performed.

\section{Inclusion criteria}

For inclusion in the trial, patients had to have a diagnosis of dementia according to the Diagnostic and Statistical Manual of Mental Disorders, Fourth Edition (DSM-IV) criteria. We diagnosed dementia subtypes based on the diagnostic criteria for Alzheimer's disease in the National Institute for Communicative Disorders and Stroke-Alzheimer's Disease and Related Disorders Association (NINCDS-ADRDA), ${ }^{19}$ the criteria for vascular dementia in the National Institute of Neurological Disorders and Stroke-Association International Pour la Recherche et I'Enseignement en Neurosciences (NINDS-AIREN) International Workshop, ${ }^{20}$ and the consensus guidelines for dementia with Lewy bodies. ${ }^{21}$ Additionally, we included patients whose total score in the Mini-Mental State Examination (MMSE) 22 was less than 19 and who had at least one symptom score of $>4$ in the Neuropsychiatric Inventory in Nursing Home Version (NPI-NH) ${ }^{23}$ subscales at the time of starting the trial. Regardless of the response or non-response received, we extracted data for all patients who accomplished treatment for 8 weeks.

\section{Exclusion criteria}

Patients were excluded from the study for any of the following criteria: participation in any other drug trial within 4 weeks of the first trial visit; known or suspected hypersensitivity to risperidone, yokukansan, or fluvoxamine; evidence of chronic and/or severe disease; and severe or unstable disease of any type that could interfere with the trial. 


\section{Treatments}

Yokukansan, TJ-54, was obtained from Tsumura Co. (Tokyo, Japan). Seven and a half grams of yokukansan extract granules contain $3.25 \mathrm{~g}$ of the following mixed drugs: $4 \mathrm{~g}$ of Japanese Pharmacopoeia (JP) Atractylodes Lancea Rhizome, $4 \mathrm{~g}$ of JP Poria Sclerotium, $3 \mathrm{~g}$ of JP Cnidium Rhizome, $3 \mathrm{~g}$ of JP Uncaria Hook, $3 \mathrm{~g}$ of Japanese Angelica Root, $2 \mathrm{~g}$ of JP Bupleurum Root and $1.5 \mathrm{~g}$ of Glycyrrhiza. These herbs are registered in the Pharmacopoeia of Japan 15th edition (2010).

Patients who met the inclusion criteria at the screening visit began a washout, during which all psychotropic medications were discontinued. The length of the washout period was at least 1 week. Zopiclone (7.5-10 mg/day) and brotizolam $(0.25 \mathrm{mg} /$ day $)$ were permitted to be taken orally for insomnia. Donepezil was also permitted if already started before trial entry. Anticholinergic medication was allowed for the treatment of extrapyramidal symptoms if a reduction in the trial medication dose was not effective. Other than these drugs, no psychotropic medication was administered during the trial.

At the first visit, patients were simply randomized to a flexible oral dosing regimen of yokukansan (2.5-7.5 g/day), risperidone ( $0.5-2.0 \mathrm{mg} /$ day), or fluvoxamine (25-200 mg/day) by the trial investigator to preserve rater-blinding (the rater was responsible for the correct conduct of psychological scales and tests, but did not know which medications were administered to which patients). These regimens were similar to the dose ranges and increment dose ranges reported in methodology for yokukansan, ${ }^{6}$ risperidone, ${ }^{24}$ and fluvoxamin ${ }^{25}$ in previous studies on elderly patients with psychotic disorders. The patients were randomized sequentially according to a computer-generated scheme.

Dose adjustments were made at the discretion of the trial investigator according to the patients' clinical responses. The doses were increased in units of $2.5 \mathrm{~g}$ /day for yokukansan, 0.5 or $1.0 \mathrm{mg}$ /day for risperidone, and 25 or $50 \mathrm{mg}$ /day for fluvoxamine. Titration to the maximal dose for each drug was permitted if the drug was tolerated by the patients according to the judgment of the investigator.

\section{Assessments}

Assessments were made at baseline and the study endpoint of 8 weeks. BPSD and cognitive functions were evaluated using the NPI-NH and MMSE, respectively.

\section{Efficacy evaluation}

The primary efficacy endpoint was the change from baseline to study endpoint in the neuropsychiatric status as measured by the NPI-NH. This instrument was developed for use by professional caregivers and evaluates 12 neuropsychiatric symptoms. Assessment by the NPI-NH is defined to rate the severity and frequency of the symptoms.

\section{Cognitive function evaluation}

The secondary endpoint was the change from baseline to study endpoint in cognitive function as measured by the MMSE.

\section{Sample collection}

As described in detail previously, ${ }^{26}$ blood was drawn from each subject every 2 weeks by venipuncture in a bloodcollection tube containing anticoagulant ethylenediamine tetra-acetic acid between 10 am and $5 \mathrm{pm}$. The tubes were immediately cooled to $4^{\circ} \mathrm{C}$ and then centrifuged at $2,000 \times g$ for 20 minutes. The plasma samples obtained were kept at $-80^{\circ} \mathrm{C}$ until analysis.

\section{Laboratory assays}

The plasma levels of homovanillic acid (HVA) and 3-methoxy-4-hydroxyphenylglycol (MHPG) were analyzed by high-performance liquid chromatography with electrochemical detection ${ }^{26-28}$ using internal standard (5-hydroxyindolecarboxylic acid) and standard addition methods.

\section{Statistical analysis}

Quantitative variables are expressed as mean \pm SD and qualitative variables are expressed as absolute value and percentage, unless otherwise noted. Comparisons of baseline demographic and clinical characteristics (sex, age, diagnosis of dementia, donepezil rate, NPI-NH, and MMSE) were investigated using the $\chi^{2}$-test or one-way analysis of variance (ANOVA) for group differences. Changes were examined for treatment effects using the Wilcoxon signedrank test to compare the baseline and endpoint values for each treatment. The Bonferroni test was used as a post hoc test for the repeated-measures ANOVA. Comparisons of individual drugs were performed by ANOVA, and Scheffe's $F$ test was used as a post hoc test. Statistical significance was defined as $P<0.05$. All analyses were performed using SPSS software version 19.0 for Macintosh (SPSS Japan, Tokyo, Japan).

\section{Results}

\section{Study participants}

Ninety patients were screened. Of these patients, eight failed to meet the inclusion criteria, by having total NPI-NH scores of $<3$. Therefore, 82 patients were included and 


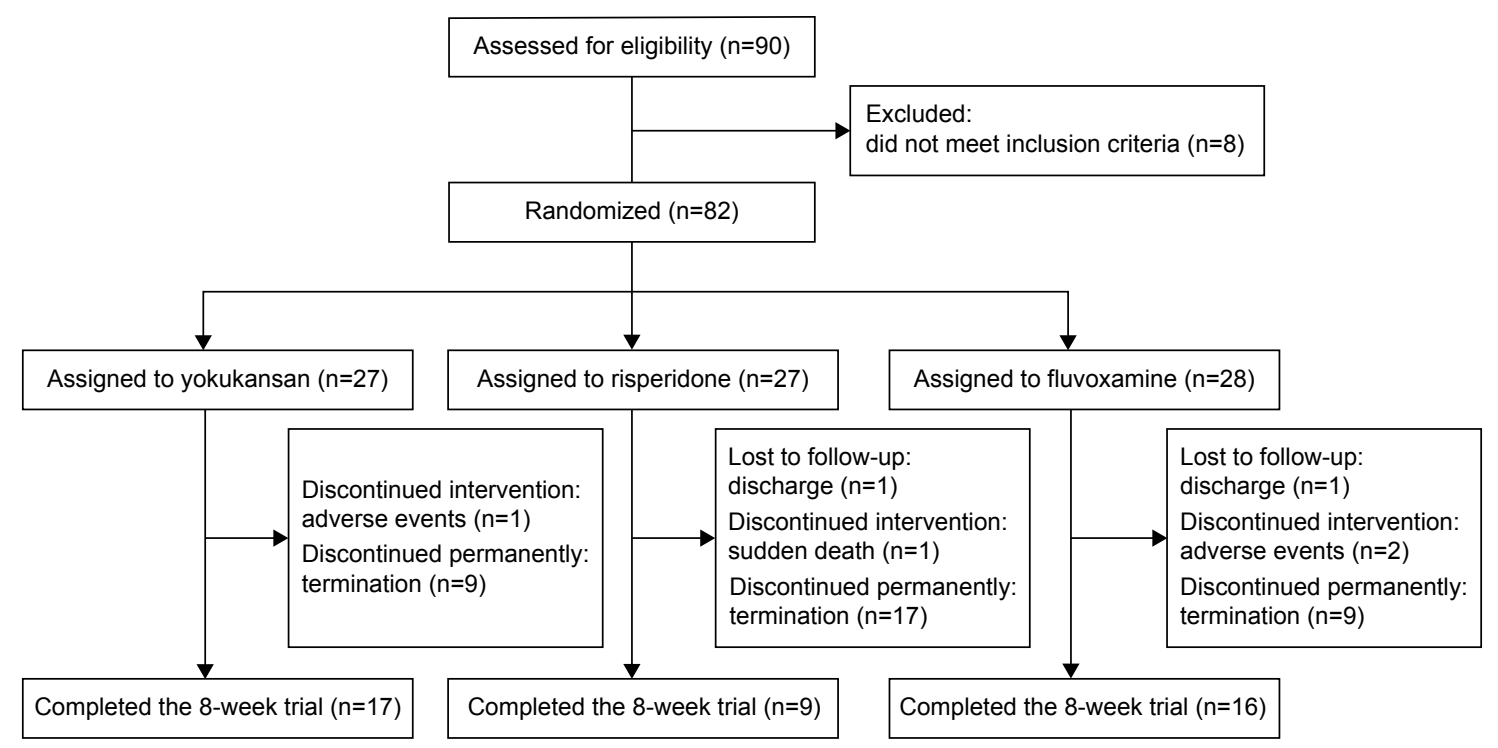

Figure I Flowchart of patient numbers throughout the trial.

treated with yokukansan, risperidone, or fluvoxamine. Forty patients (yokukansan: 10; risperidone: 18; fluvoxamine: 12) did not complete the follow-up component of the study. These participants discontinued for the following reasons. Sixteen patients (yokukansan: 3; risperidone: 9; fluvoxamine: 4) did not continue the 8-week trial as they were discharged because of improvement, family reasons, or entrance to facility. One patient on risperidone died suddenly. Thirteen patients discontinued the trial because of deterioration of psychiatric symptoms (yokukansan: 5; risperidone: 3; fluvoxamine: 5). Thirteen patients discontinued the trial because of somatic symptoms (yokukansan: 3; risperidone: 5; fluvoxamine: 3 ), such as fracture, cerebral hemorrhage, dysphagia, oversedation, asthma, pneumonia, bilateral subdural hematoma, or fever. Thus, 42 patients (yokukansan: 17; risperidone: 9; fluvoxamine: 16) completed 8 weeks of treatment in the study (Figure 1) and were analyzed.

\section{Patient characteristics}

The baseline characteristics for the intention-to-treat population are shown in Table 1. No significant differences among the yokukansan, risperidone, and fluvoxamine groups were found with respect to sex, age, distribution of dementia diagnosis, and concomitant use of donepezil $(P>0.05)$. Furthermore, no significant group differences among the three drugs

Table I Demographic and baseline characteristics of the intention-to-treat population

\begin{tabular}{|c|c|c|c|c|}
\hline Characteristics & Risperidone $n=9$ & Yokukansan $n=17$ & Fluvoxamine $n=16$ & $P$-value \\
\hline \multicolumn{5}{|l|}{ Sex, n (\%) } \\
\hline Male & $3(33.3)$ & $3(17.6)$ & $6(37.5)$ & 0.423 \\
\hline Female & $6(66.7)$ & $14(82.4)$ & $10(62.5)$ & \\
\hline Mean age, years (SD) & $80.2(9.0)$ & $83.6(6.0)$ & $83.9(4.9)$ & 0.405 \\
\hline \multicolumn{5}{|l|}{ Diagnosis, n (\%) } \\
\hline$A D$ & $6(66.7)$ & 14 (82.4) & II (68.8) & 0.367 \\
\hline VD & $3(33.3)$ & $3(17.6)$ & $3(18.8)$ & \\
\hline DLB & $0(0.0)$ & $0(0.0)$ & $2(12.5)$ & \\
\hline \multicolumn{5}{|l|}{ Donepezil, n (\%) } \\
\hline Yes & $6(66.7)$ & $13(76.5)$ & I5 (93.8) & 0.211 \\
\hline No & $3(33.3)$ & $4(23.5)$ & I (6.3) & \\
\hline \multicolumn{5}{|l|}{ Assessments (points) } \\
\hline NPI-NH (SD) & $29.4(17.1)$ & $26.1(14.3)$ & $22.0(12.2)$ & 0.413 \\
\hline MMSE (SD) & $3.8(4.5)$ & $2.9(3.6)$ & $4.3(5.5)$ & 0.506 \\
\hline
\end{tabular}

Abbreviations: AD, Alzheimer's disease; VD, vascular dementia; DLB, dementia with Lewy bodies; MMSE , Mini-Mental State Examination; NPI-NH, Neuropsychiatric Inventory in Nursing Home Version; SD, standard deviation. 
Table 2 Mean changes in biological markers

\begin{tabular}{llllllll}
\hline Variables & $\mathbf{n}$ & Baseline & 2 weeks & 4 weeks & 6 weeks & 8 weeks & P-value \\
\hline $\begin{array}{l}\text { MHPG }(\mathrm{ng} / \mathrm{mL}) \\
\text { Risperidone }\end{array}$ & 9 & $13.7(6.4)$ & $14.4(5.2)$ & $12.7(4.3)$ & $14.9(4.5)$ & $13.1(4.6)$ & 0.435 \\
$\begin{array}{l}\text { Yokukansan } \\
\text { Fluvoxamine }\end{array}$ & 17 & $13.4(6.7)$ & $11.0(3.4)$ & $11.8(4.1)$ & $12.7(3.8)$ & $13.0(4.8)$ & 0.191 \\
HVA (ng/mL) & 16 & $12.1(4.6)$ & $12.1(4.8)$ & $11.3(4.6)$ & $10.7(3.2)$ & $10.4(2.5)$ & 0.313 \\
$\quad$ Risperidone & 9 & $12.8(5.4)$ & $14.6(6.3)$ & $12.2(5.5)$ & $12.8(4.0)$ & $13.6(3.5)$ & 0.548 \\
$\quad \begin{array}{l}\text { Yokukansan } \\
\text { Fluvoxamine }\end{array}$ & 17 & $17.1(7.6)$ & $12.4(7.7)$ & $13.3(6.0)$ & $11.2(3.4)$ & $12.2(6.8)$ & $0.006 * *$ \\
\hline
\end{tabular}

Notes: Quantitative variables expressed as mean (standard deviation). Changes were examined for treatment effects using the repeated-measures ANOVA ( $* * P<0.0 \mathrm{I}$ ). Abbreviations: ANOVA, analysis of variance; HVA, homovanillic acid; MHPG, 3-methoxy-4-hydroxyphenylglycol.

were found with respect to assessments of $\operatorname{BPSD}(P=0.413)$ and cognitive function $(P=0.506)$ at baseline. Additionally, ECG and laboratory data including complete blood count, chemistry, urinalysis, thyroid function, vitamin B12, and folate levels were mostly within the normal range in all patients.

\section{Treatments}

As described in detail previously, ${ }^{9}$ medication was based on a flexible-dose regimen, that is, the dose of trial medication was adjusted according to the patients' clinical response and tolerability at the discretion of the trial investigator. In this study, the mean doses at 8 weeks for yokukansan, risperidone, and fluvoxamine were $6.32 \pm 2.19 \mathrm{~g} /$ day, $1.17 \pm 0.71 \mathrm{mg} /$ day, and $87.5 \pm 64.5 \mathrm{mg} /$ day, respectively.

\section{Assessments}

All three drugs significantly reduced the NPI-NH total scores between baseline and study endpoint. The total NPI-NH scores decreased from $26.1 \pm 14.3$ to $12.5 \pm 10.6$ with yokukansan $(P=0.002), 29.4 \pm 17.1$ to $16.6 \pm 9.5$ with risperidone $(P=0.044)$, and $22.0 \pm 12.2$ to $11.4 \pm 10.6$ with fluvoxamine $(P=0.003)$. No significant differences among the yokukansan, risperidone, and fluvoxamine groups were found with respect to the assessment of BPSD at baseline $(P=0.413)$ (Table 1).

\section{Assessment of cognitive function (MMSE)}

No significant changes in the MMSE scores were observed between baseline and study endpoint for all drugs. Specifically, the MMSE scores changed little from 2.9 \pm 3.6 to $4.1 \pm 4.5$ with yokukansan $(P=0.057), 3.8 \pm 4.5$ to $2.9 \pm 4.3$ with risperidone $(P=0.179)$, and $4.3 \pm 5.4$ to $3.6 \pm 5.8$ with fluvoxamine $(P=0.291)$. No significant differences among the yokukansan, risperidone, and fluvoxamine groups were found with respect to the assessment of cognitive function at baseline $(P=0.506)$ (Table 1$)$.

\section{Biological markers HVA at baseline}

The HVA levels at baseline were as follows: $17.1 \pm 7.6 \mathrm{ng} /$ $\mathrm{mL}$ for the yokukansan group $(\mathrm{n}=17), 12.8 \pm 5.4 \mathrm{ng} / \mathrm{mL}$ for the risperidone group $(n=9)$, and 13.9 \pm 3.9 for the fluvoxamine group $(n=16) \mathrm{ng} / \mathrm{mL}$. No significant differences were found among the yokukansan, risperidone, and fluvoxamine groups with respect to HVA levels at baseline $(P=0.154)$. Including the dropout participants, the HVA levels at baseline were as follows: $17.2 \pm 8.1 \mathrm{ng} / \mathrm{mL}$ for the yokukansan group ( $\mathrm{n}=27), 17.6 \pm 14.2 \mathrm{ng} / \mathrm{mL}$ for the risperidone group $(\mathrm{n}=27)$, and $15.2 \pm 6.5 \mathrm{ng} / \mathrm{mL}$ for the fluvoxamine group $(n=28)$. No significant differences were found between the three groups in terms of the HVA levels at baseline when the dropout patients were included $(P=0.638)$. The HVA levels at baseline in the dropout patients were as follows: $17.4 \pm 9.4 \mathrm{ng} / \mathrm{mL}$ in the yokukansan group $(\mathrm{n}=10), 20.0 \pm 16.7$ $\mathrm{ng} / \mathrm{mL}$ in the risperidone group $(\mathrm{n}=18)$, and $16.9 \pm 8.8 \mathrm{ng} /$ $\mathrm{mL}$ in the fluvoxamine group $(\mathrm{n}=12) \mathrm{ng} / \mathrm{mL}$. No significant differences were found between the completed group and the dropout group in terms of the HVA levels at baseline in each medication group; (Mann-Whitney $U$-test; $P=0.941$ for yokukansan, $P=0.053$ for risperidone, and $P=0.599$ for fluvoxamine).

\section{Changes of HVA (dopamine metabolite)}

The patients in the yokukansan group had significantly decreased plasma HVA levels during the entire period (repeated-measures ANOVA: $P=0.006$ ) (Table 2 and Figure 2). Specifically, the plasma HVA levels differed significantly from baseline within the yokukansan group at 6 weeks $(P=0.037)$ and 8 weeks $(P=0.036)$ (repeated-measures ANOVA, post hoc Bonferroni multiple comparison). However, the patients in the risperidone and fluvoxamine groups exhibited no significant changes in plasma HVA from baseline (repeated-measures ANOVA: $P=0.548$ for risperidone and $P=0.414$ for fluvoxamine). 


\section{Changes of MHPG (noradrenaline metabolite)}

The patients in all the three groups exhibited no significant changes in plasma MHPG during the entire period (repeatedmeasures ANOVA: $P=0.191$ for yokukansan; $P=0.435$ for risperidone; and $P=0.313$ for fluvoxamine) (Table 2 and Figure 2). No significant differences among the yokukansan, risperidone, and fluvoxamine groups were found with respect to plasma MHPG levels at baseline $(P=0.748)$.

\section{Discussion}

We previously reported that risperidone, yokukansan, and fluvoxamine showed equal efficacy in the treatment of BPSD in elderly patients. ${ }^{9}$ Although all the three drugs were effective in the treatment of BPSD, the specific mechanisms modified by each drug remain unclear. The mechanisms of the individual drugs are as follows. The neuropharmacological properties of risperidone are primarily exerted through the antagonism of both serotonin 5-HT2 and dopamine D2 receptors. ${ }^{17}$ Fluvoxamine is chemically unrelated to other currently available antidepressants. ${ }^{29}$ The neuropharmacological properties of fluvoxamine are suggested to include its serotonin reuptake inhibitor effects. The neuropharmacological properties of yokukansan are suggested to include its serotonin $1 \mathrm{~A}$ receptor partial agonistic effects ${ }^{30,31}$ and serotonin 2 receptor downregulatory effects. ${ }^{14}$ Thus, the neuropharmacological properties of all the three drugs are characterized by potent central monoamines. Therefore, we aimed to clarify the changes in monoamines caused by the treatment of each drug in patients with BPSD. In this study, the BPSD data from patients who completed 8 weeks of treatment with the three drugs were analyzed. In accordance with a previous study using the last-observation-carried-forward method, ${ }^{9}$

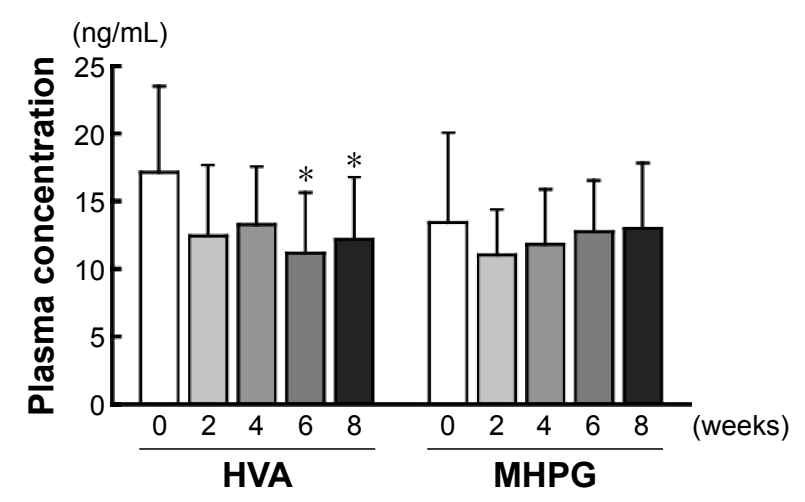

Figure 2 Courses of biological markers in the yokukansan group.

Note: Changes were examined for treatment effects using the Wilcoxon signedrank test to compare baseline values $(* P<0.05)$.

Abbreviations: HVA, homovanillic acid (metabolite of dopamine); MHPG, 3-methoxy-4-hydroxyphenylglycol (metabolite of noradrenaline). we found that all the three drugs were found to be effective in the treatment of BPSD.

Yokukansan significantly decreased HVA, reflecting dopamine metabolism. When yokukansan was screened for the presence of antipsychotic agents, Pengsuparp et al identified geissoschizine methyl ether (GM), an indole alkaloid..$^{32} \mathrm{GM}$ was demonstrated to act as a partial agonist for dopamine D2 receptors with similar intrinsic activity to aripiprazole. ${ }^{33}$ Dopamine D2 receptors are important targets for pharmaceutical agents used in the treatment of schizophrenia. Miyaoka et a ${ }^{34}$ reported that yokukansan is an efficacious treatment for psychosis in schizophrenia. Interestingly, aripiprazole, which acts as a partial D2 agonist similar to GM, significantly decreased HVA, reflecting dopamine metabolite levels. ${ }^{35}$ In this study, the decreased HVA induced by yokukansan may be due to the constituents of yokukansan such as GM partially inhibiting D2 receptors, resulting in significantly decreased dopamine metabolites in a time-dependent manner. In other words, in BPSD, the mechanism of yokukansan may involve stabilization of excessive dopamine. In this study, we first found that yokukansan decreases the dopamine metabolite, HVA, in treatment for BPSD.

Many previous studies have demonstrated that risperidone reduces neuropsychiatric symptoms in elderly patients with dementia..$^{2436-39}$ Risperidone, an atypical antipsychotic, has proven to be effective in the treatment of patients with positive symptoms of schizophrenia as demonstrated by many reports. ${ }^{40}$ It is widely known that the neuropharmacological properties of risperidone are primarily exerted through antagonism of dopamine D2 receptors ${ }^{17}$ and that favorable responses for schizophrenia in the acute phase are associated with elevated pretreatment HVA levels and changes in HVA. ${ }^{41,42}$ Patients in the risperidone group exhibited no significant change in HVA from baseline. As the underlying reason for this finding, although the average dose of risperidone for schizophrenia was $3.8 \mathrm{mg} /$ day in a previous study, ${ }^{42}$ the average dose of risperidone in this study was $1.2 \mathrm{mg} /$ day. In other studies, the average dosages of risperidone for BPSD were $0.9 \mathrm{mg} /$ day, ${ }^{36} 1.0 \mathrm{mg} /$ day, ${ }^{37}$ and $0.8 \mathrm{mg} /$ day ${ }^{24} \mathrm{In}$ our study, low-dose risperidone was fully effective in treating BPSD in elderly patients. Therefore, it is suggested that lowdose risperidone fails to induce changes in HVA. A metaanalysis of randomized placebo-controlled trials suggested a significantly higher risk of death with atypical antipsychotic drug treatment for dementia than with placebo treatment. ${ }^{43}$ Furthermore, in 2005, the Food and Drug Administration of the United States requested a health advisory warning on the prescription information for all atypical antipsychotics stating 
that there is an increased risk of mortality in elderly patients with dementia-related psychosis taking these drugs compared with placebo. ${ }^{44}$ Attempts may be made to avoid antipsychotic drugs in treating BPSD as much as possible.

A double-blind, placebo-controlled study of citalopram, an SSRI, demonstrated its efficacy for behavioral disturbances in the treatment of BPSD. ${ }^{11} \mathrm{We}$ prescribed fluvoxamine, an SSRI, to treat BPSD in three patients with dementia and reported its efficacy. ${ }^{18}$ Furthermore, a randomized controlled study of fluvoxamine demonstrated its efficacy for BPSD. ${ }^{9}$ In the present study, both HVA and MHPG tended to show decreases between baseline and study endpoint, but the differences were not statistically significant (Wilcoxon signed-rank, $P>0.05$ ). Because it is known that lesions in serotonin neurons result in increased dopamine neuronal activity in the ventral tegmental area, it has been proposed that enhanced serotonin levels inhibit dopamine neurons. ${ }^{45}$ Citalopram, the same kind of SSRI as fluvoxamine, induces the inhibition of noradrenaline neuronal activity by a mechanism involving serotonin-2A receptors. ${ }^{46}$ Through this mechanism, serotonin is increased in the synaptic cleft by fluvoxamine, followed by the inhibition of neuronal firing of dopamine and noradrenaline, resulting in the release of dopamine and a decrease in noradrenaline. Thus, the efficacy of fluvoxamine for BPSD may be suggested to involve increased serotonin in the synaptic cleft that induces inhibition of dopamine and noradrenaline. The duration of the trial was set at 8 weeks according to the original protocol. The number of participants who dropped out of the study was an unpredictable factor. Although no significant HVA differences were found between the completed group and the dropout group, the number of dropouts might influence the statistical power of the study. Although sex is not significant among the groups, the yokukansan group appeared to have a higher proportion of females than was in the other groups. However, the characteristics such as age, HVA, or MHPG showed no significant differences regarding sex at baseline. Not only dopamine but also serotonin has a role in the treatment of BPSD. A relationship between the 5-hydroxytryptamine (5-HT) transporter and BPSD was demonstrated in Alzheimer's disease. ${ }^{47,48}$ It was reported that 5-HT2A inverse agonists, pimavanserin, suppressed psychosis-like behaviors in a rodent model of Alzheimer's disease. ${ }^{49}$ Pharmacological profiles of risperidone may show a relatively higher affinity for 5-HT2 receptors than $\mathrm{D} 2$ receptors. Thus, risperidone may improve BPSD by blocking 5-HT2 receptors. ${ }^{50}$ Pharmacological profiles of yokukansan have shown that yokukansan acted as a partial agonist at the 5-HT1A receptors ${ }^{30,31}$ and showed a suppressive effect through the downregulation of the 5-HT2A receptors. ${ }^{14}$ Furthermore, it was also reported that yokukansan activated the astrocytic glutamate transporter and showed a neuroprotective effect against glutamate-mediated excitotoxicity. ${ }^{51}$ More large-scale design, including placebo group, or a wide range of several neurotransmitter studies are needed to understand the mechanism of yokukansan for the treatment of BPSD.

\section{Conclusion}

In this study, we first found that yokukansan decreases the dopamine metabolite, HVA, in treatment for BPSD. Furthermore, catecholamines, especially dopamine, are indicated to show the possible pathophysiology of BPSD. In future, the pathophysiology of BPSD will hopefully become clear from a biological viewpoint, and hence treatments for BPSD can be established based on biological markers.

\section{Acknowledgments}

We thank the study participants, without whom this study would not have been accomplished, as well as the following psychiatrists of Sato Hospital, Koutokukai, for their participation in this study: Kazuo Kenmi, MD, PhD; Asao Hasegawa, MD; Masaaki Mitomo, MD; Tadanori Kumasaka, MD, PhD; Tomotaka Suzuki, MD; Yukihiro Takeuchi, MD, PhD; Hiroshi Yamamoto, MD; Daisuke Saito, MD; Kenji Takeyoshi, MD; and Kohei Koizumi, MD.

\section{Disclosure}

This study was supported by funding from Sato Hospital, Koutokukai. The study did not receive funding from any pharmaceutical corporations. The authors report no biomedical financial interests or potential conflicts of interest in this work.

\section{References}

1. Finkel SI. Behavioral and psychologic symptoms of dementia. Clin Geriatr Med. 2003;19(4):799-824.

2. Lawlor BA. Behavioral and psychological symptoms in dementia: the role of atypical antipsychotics. J Clin Psychiatry. 2004; 65(Suppl 11):5-10.

3. Chiu MJ, Chen TF, Yip PK, Hua MS, Tang LY. Behavioral and psychologic symptoms in different types of dementia. J Formo Med Assoc. 2006;105(7):556-562.

4. Hayashi $Y$, Ishida $Y$, Inoue T, et al. Treatment of behavioral and psychological symptoms of Alzheimer-type dementia with Yokukansan in clinical practice. Prog Neuropsychopharmacol Biol Psychiatry. 2010; 34(3):541-545.

5. Iwasaki K, Kosaka K, Mori H, et al. Open label trial to evaluate the efficacy and safety of Yokukansan, a traditional Asian medicine, in dementia with Lewy bodies. $J$ Am Geriatr Soc. 2011;59(5): 936-938. 
6. Iwasaki K, Satoh-Nakagawa T, Maruyama M, et al. A randomized, observer-blind, controlled trial of the traditional Chinese medicine YiGan San for improvement of behavioral and psychological symptoms and activities of daily living in dementia patients. J Clin Psychiatry. 2005;66(2):248-252.

7. Monji A, Takita M, Samejima T, et al. Effect of yokukansan on the behavioral and psychological symptoms of dementia in elderly patients with Alzheimer's disease. Prog Neuropsychopharmacol Biol Psychiatry. 2009;33(2):308-311.

8. Nagata K, Yokoyama E, Yamazaki T, et al. Effects of yokukansan on behavioral and psychological symptoms of vascular dementia: an openlabel trial. Phytomedicine. 2012;19(6):524-528.

9. Teranishi M, Kurita M, Nishino S, et al. Efficacy and tolerability of risperidone, yokukansan, and fluvoxamine for the treatment of behavioral and psychological symptoms of dementia: a blinded, randomized trial. J Clin Psychopharmacol. 2013;33(5):600-607.

10. Schneider LS, Pollock VE, Lyness SA. A metaanalysis of controlled trials of neuroleptic treatment in dementia. J Am Geriatr Soc. 1990;38(5):553-563.

11. Pollock BG, Mulsant BH, Rosen J, et al. Comparison of citalopram, perphenazine, and placebo for the acute treatment of psychosis and behavioral disturbances in hospitalized, demented patients. $\mathrm{Am}$ $J$ Psychiatry. 2002;159(3):460-465.

12. Lanari A, Amenta F, Silvestrelli G, Tomassoni D, Parnetti L. Neurotransmitter deficits in behavioural and psychological symptoms of Alzheimer's disease. Mech Ageing Dev. 2006;127(2):158-165.

13. Mizukami K, Asada T, Kinoshita T, et al. A randomized cross-over study of a traditional Japanese medicine (kampo), yokukansan, in the treatment of the behavioural and psychological symptoms of dementia. Int J Neuropsychopharmacol. 2009;12(2):191-199.

14. Egashira N, Iwasaki K, Ishibashi A, et al. Repeated administration of Yokukansan inhibits DOI-induced head-twitch response and decreases expression of 5-hydroxytryptamine (5-HT)2A receptors in the prefrontal cortex. Prog Neuropsychopharmacol Biol Psychiatry. 2008; 32(6):1516-1520.

15. Rojas-Fernandez CH, Lanctot KL, Allen DD, MacKnight C. Pharmacotherapy of behavioral and psychological symptoms of dementia: time for a different paradigm? Pharmacotherapy. 2001;21(1):74-102.

16. Schneider LS, Dagerman K, Insel PS. Efficacy and adverse effects of atypical antipsychotics for dementia: meta-analysis of randomized, placebo-controlled trials. Am J Geriatr Psychiatry. 2006;14(3): 191-210.

17. Creese I, Burt DR, Snyder SH. Dopamine receptor binding predicts clinical and pharmacological potencies of antischizophrenic drugs. Science. 1976;192(4238):481-483.

18. Kurita M, Sato T, Nishino S, et al. Effects of fluvoxamine on behavioral and psychological symptoms of dementia in Alzheimer's disease: a report of three cases. Fukushima J Med Sci. 2006;52(2): $143-148$.

19. McKhann G, Drachman D, Folstein M, Katzman R, Price D, Stadlan EM. Clinical diagnosis of Alzheimer's disease: report of the NINCDSADRDA Work Group under the auspices of Department of Health and Human Services Task Force on Alzheimer's Disease. Neurology. 1984;34(7):939-944.

20. Roman GC, Tatemichi TK, Erkinjuntti T, et al. Vascular dementia: diagnostic criteria for research studies. Report of the NINDS-AIREN International Workshop. Neurology. 1993;43(2):250-260.

21. McKeith IG, Galasko D, Kosaka K, et al. Consensus guidelines for the clinical and pathologic diagnosis of dementia with Lewy bodies (DLB): report of the consortium on DLB international workshop. Neurology. 1996;47(5):1113-1124.

22. Folstein MF, Folstein SE, McHugh PR. "Mini-mental state". A practical method for grading the cognitive state of patients for the clinician. J Psychiatr Res. 1975;12(3):189-198.

23. Wood S, Cummings JL, Hsu MA, et al. The use of the neuropsychiatric inventory in nursing home residents. Characterization and measurement. Am J Geriatr Psychiatry. 2000;8(1):75-83.
24. Rocca P, Marino F, Montemagni C, Perrone D, Bogetto F. Risperidone, olanzapine and quetiapine in the treatment of behavioral and psychological symptoms in patients with Alzheimer's disease: preliminary findings from a naturalistic, retrospective study. Psychiatry Clin Neurosci. 2007;61(6):622-629.

25. Phanjoo AL, Wonnacott S, Hodgson A. Double-blind comparative multicentre study of fluvoxamine and mianserin in the treatment of major depressive episode in elderly people. Acta Psychiatr Scand. 1991;83(6): 476-479.

26. Kurita M, Nishino S, Numata Y, Okubo Y, Sato T. The noradrenaline metabolite MHPG is a candidate biomarker from the manic to the remission state in bipolar disorder I: a clinical naturalistic study. PLoS One. 2012;9(6):e100634.

27. Yeung PK, Buckley SJ, Pedder SC, Dingemanse J. Determination of 3,4dihydroxyphenylacetic acid and 5-hydroxyindoleacetic acid in human plasma by a simple and rapid high-performance liquid chromatography assay. J Pharma Sci. 1996;85(4):451-453.

28. Yoshimura R, Nakamura J, Shinkai K, et al. An open study of risperidone liquid in the acute phase of schizophrenia. Hum Psychopharmacol. 2005;20(4):243-248.

29. Pacher P, Kecskemeti V. Trends in the development of new antidepressants. Is there a light at the end of the tunnel? Curr Med Chem. 2004;11(7): 925-943.

30. Kanno H, Sekiguchi K, Yamaguchi T, et al. Effect of yokukansan, a traditional Japanese medicine, on social and aggressive behaviour of para-chloroamphetamine-injected rats. J Pharm Pharmacol. 2009; 61(9):1249-1256.

31. Terawaki K, Ikarashi Y, Sekiguchi K, Nakai Y, Kase Y. Partial agonistic effect of yokukansan on human recombinant serotonin 1A receptors expressed in the membranes of Chinese hamster ovary cells. J Ethnopharmacol. 2010;127(2):306-312.

32. Pengsuparp T, Indra B, Nakagawasai O, et al. Pharmacological studies of geissoschizine methyl ether, isolated from Uncaria sinensis Oliv., in the central nervous system. Eur J Pharmacol. 2001;425(3): 211-218.

33. Ueda T, Ugawa S, Ishida Y, Shimada S. Geissoschizine methyl ether has third-generation antipsychotic-like actions at the dopamine and serotonin receptors. Eur J Pharmacol. 2011;671(1-3):79-86.

34. Miyaoka T, Wake R, Furuya M, et al. Yokukansan (TJ-54) for treatment of very-late-onset schizophrenia-like psychosis: an open-label study. Phytomedicine. 2013;20(7):654-658.

35. Yoshimura R, Ueda N, Hori H, Ikenouchi-Sugita A, Umene-Nakano W, Nakamura J. Different patterns of longitudinal changes in plasma levels of catecholamine metabolites and brain-derived neurotrophic factor after administration of atypical antipsychotics in first episode untreated schizophrenic patients. World J Biol Psychiatry. 2010; 11(2 Pt 2):256-261.

36. Rainer M, Haushofer M, Pfolz H, Struhal C, Wick W. Quetiapine versus risperidone in elderly patients with behavioural and psychological symptoms of dementia: efficacy, safety and cognitive function. Eur Psychiatry. 2007;22(6):395-403.

37. Brodaty H, Ames D, Snowdon J, et al. A randomized placebo-controlled trial of risperidone for the treatment of aggression, agitation, and psychosis of dementia. J Clin Psychiatry. 2003;64(2):134-143.

38. Ellingrod VL, Schultz SK, Ekstam-Smith K, Kutscher E, Turvey C, Arndt S. Comparison of risperidone with olanzapine in elderly patients with dementia and psychosis. Pharmacotherapy. 2002;22(1):1-5.

39. Katz I, de Deyn PP, Mintzer J, Greenspan A, Zhu Y, Brodaty H. The efficacy and safety of risperidone in the treatment of psychosis of Alzheimer's disease and mixed dementia: a meta-analysis of 4 placebo-controlled clinical trials. Int J Geriatr Psychiatry. 2007;22(5): 475-484.

40. Umbricht D, Kane JM. Risperidone: efficacy and safety. Schizophr Bull. 1995;21(4):593-606.

41. Mazure CM, Nelson JC, Jatlow PI, Bowers MB. Plasma free homovanillic acid (HVA) as a predictor of clinical response in acute psychosis. Biol Psychiatry. 1991;30(5):475-482. 
42. Yoshimura R, Ueda N, Shinkai K, Nakamura J. Plasma levels of homovanillic acid and the response to risperidone in first episode untreated acute schizophrenia. Int Clin Psychopharmacol. 2003;18(2):107-111.

43. Schneider LS, Dagerman KS, Insel P. Risk of death with atypical antipsychotic drug treatment for dementia: meta-analysis of randomized placebo-controlled trials. JAMA. 2005;294(15):1934-1943.

44. FDA. Public Health Advisory: Deaths with Antipsychotics in Elderly Patients with Behavioral Disturbances. Silver Spring, MD: FDA; 2005. Available from: http://www.fda.gov/drugs/drugsafety/postmarketdrugsafetyinformationforpatientsandproviders/ucm053171. Accessed November 26, 2015.

45. Guiard BP, El Mansari M, Merali Z, Blier P. Functional interactions between dopamine, serotonin and norepinephrine neurons: an in-vivo electrophysiological study in rats with monoaminergic lesions. Int $J$ Neuropsychopharmacol. 2008;11(5):625-639.

46. Dremencov E, El Mansari M, Blier P. Noradrenergic augmentation of escitalopram response by risperidone: electrophysiologic studies in the rat brain. Biol Psychiatry. 2007;61(5):671-678.
47. Assal F, Alarcon M, Solomon EC, Masterman D, Geschwind DH, Cummings JL. Association of the serotonin transporter and receptor gene polymorphisms in neuropsychiatric symptoms in Alzheimer disease. Arch Neurol. 2004;61(8):1249-1253.

48. Pritchard AL, Pritchard CW, Bentham P, Lendon CL. Role of serotonin transporter polymorphisms in the behavioural and psychological symptoms in probable Alzheimer disease patients. Dement Geriatr Cogn Dis. 2007;24(3):201-206.

49. Price DL, Bonhaus DW, McFarland K. Pimavanserin, a 5-HT2A receptor inverse agonist, reverses psychosis-like behaviors in a rodent model of Alzheimer's disease. Behav Pharmacol. 2012;23(4):426-433.

50. Kapur S, Remington G, Zipursky RB, Wilson AA, Houle S. The D2 dopamine receptor occupancy of risperidone and its relationship to extrapyramidal symptoms: a PET study. Life Sci. 1995;57(10):PL103-PL107.

51. Kawakami Z, Kanno H, Ueki T, et al. Neuroprotective effects of yokukansan, a traditional Japanese medicine, on glutamate-mediated excitotoxicity in cultured cells. Neuroscience. 2009;159(4):1397-1407.
Neuropsychiatric Disease and Treatment

\section{Publish your work in this journal}

Neuropsychiatric Disease and Treatment is an international, peerreviewed journal of clinical therapeutics and pharmacology focusing on concise rapid reporting of clinical or pre-clinical studies on a range of neuropsychiatric and neurological disorders. This journal is indexed on PubMed Central, the 'PsycINFO' database and CAS,

\section{Dovepress}

and is the official journal of The International Neuropsychiatric Association (INA). The manuscript management system is completely online and includes a very quick and fair peer-review system, which is all easy to use. Visit http://www.dovepress.com/testimonials.php to read real quotes from published authors.

Submit your manuscript here: http://www.dovepress.com/neuropsychiatric-disease-and-treatment-journal 